\title{
A Case of Eccrine Porocarcinoma, Located in the Breast: The Pitfalls Reviewed
}

\author{
Chris Monten*, Jolien Berwouts, Liv Veldeman, David Creytens and Geert Braems
}

Department of Medicine and Health Sciences, Ghent University, Belgium

*Corresponding author: Chris Monten, Department of Medicine and Health Sciences, Ghent University, Belgium, Tel: +32 93310101 ; Fax: 972-4-854-2898; E-mail: chris.monten@uzgent.be

Rec Date: December 24, 2017, Acc Date: December 30, 2017, Pub Date: December 31, 2017

Citation: Monten C, Berwouts J, Veldeman L, Creytens D, Braems G (2017) Endoscopic Biological Glue Injection for Treating Multiple Staple-Line Leaks and Dehiscence Following Laparoscopic Sleeve Gastrectomy - Case Report and Review of The Literature. Med Case Rep Vol.4 No. S1:004.

\section{Abstract}

Evidence on eccrine porocarcinoma (EPC) was revised in response to the case of a 74-year old patient, presenting with an EPC located in the breast and initially treated with local excision only. She was referred to our center after loco-regional recurrence, but re-excision with lymphadenectomy and adjuvant radiotherapy to a dose of $66 \mathrm{~Gy}$ failed to obtain loco-regional control.

EPC is a rare disease, occurring in $0.005-0.01 \%$ of all skin cancers. The probability of misdiagnosis is high, especially in case of an unusual primary site of tumour location. Clinical and histological diagnosis encompasses a large list of differential diagnoses. Treatment primarily consists of surgery, with lymphadenectomy in case of unfavorable characteristics. No evidence exists for adjuvant systemic or loco-regional treatment.

Keywords: Eccrine Porocarcinoma (EPC); Breast cancer; Skin cancer; Surgery; Radiotherapy; Pathology; PET-CT

\section{Introduction}

A 74-year old patient, diagnosed with an eccrine porocarcinoma (EPC), primarily localized in the breast, was referred to our hospital.

Two years earlier she underwent a lumpectomy for a polypoid nodule in the right breast at her local hospital. Histopathology revealed an eccrine carcinoma, completely resected. As the axilla was clinically negative on palpation and ultrasonography, no lymphadenectomy was performed.

At the time of referral, the patient had already consulted her surgeon due to a painful rash and purple nodules in the superior external quadrant of the right breast. The lesions were re-resected and histopathological examination confirmed relapse of eccrine porocarcinoma, largest diameter $45 \mathrm{~mm}$. Resection margins were free, but lymphovascular invasion was present. Neither preoperative staging nor lymphadenectomy had been performed. Hormonal receptors were not analysed.
Because of this disturbing histology, a mammography and ultrasound were performed postoperatively, revealing an enlarged lymph node in the right axilla. Fine needle aspiration confirmed presence of metastatic carcinoma, compatible with EPC. Postoperative computer tomography (CT) thorax disclosed a large axillary mass, invading the pectoral muscle, with enlarged lymph nodes higher up in the axilla (level 2).

On fluorodeoxyglucose (FDG) positron emission tomography (PET)-CT the axillary mass was intensely FDG-avid, as were three axillary lymph nodes, posterior to the mass (Figure 1). On CT, two small pulmonary nodules were visible in the right upper lobe. These lesions were not FDG-avid, but follow-up was advised since PET is less sensitive for small lesions [1]. In view of these findings, the patient was referred to our centre for further investigation and treatment. An additionial bone scan did not reveal other lesions. Magnetic resonance imaging (MRI) excluded tumour-localization in the breast.

In multidisciplinary concertation, the tumour was considered to be still loco-regional (making abstraction of the pulmonary micronodules). The option of completion surgery followed by adjuvant radiotherapy was discussed with the patient. As she opted for maximal chances to obtain locoregional control, a wide resection of the residual mass was performed in combination with axillary lymphadenectomy. All skin nodules were resected. Dissection proved difficult because of strong adhesion of the tumour to surrounding tissue. Because the tumour enveloped the axillary vene it had to be transected and extensive clipping of branches of the axillary vein was needed. Immediate postoperative haemorrhage required revision of the wound to obtain haemostasis. Postoperative care was complicated due to a wound infection, which was successfully treated with antibiotics.

Histopathology reported an infiltrative, morphologically high-grade tumour connected to the epidermis with partial lobular growth architecture, compatible with the diagnosis of EPC, 'infiltrative subtype', with diameter exceeding $90 \mathrm{~mm}$ (Figure 2). The tumour had an obvious endophytic growth pattern with invasion of the deep reticular dermis and subcutis (Figures 2a-2c). Ductal differentiation was observed and the presence of ducts and intracytoplasmic lumina (Figures $\mathbf{2 d - 2 f}$ ) was highlighted with the histochemical PAS staining and the immunohistochemical EMA and CEA stainings. The tumour cells showed nuclear pleomorphism and conspicuous mitotic 
activity (Figure 2e). Areas of comedo type necrosis (Figure $\mathbf{2 d}$ ) and lymphovascular invasion (Figure $\mathbf{2 g}$ ) were observed. Foci of squamous differentiation were present. Immunohistochemistry showed tumour positivity for p63, CK5 and EMA. Estrogen and progesterone receptors and Her2Neu were negative in the tumour. P40 stained only weakly, CK7 and GATA 3 were negative. Margins were clear of tumour.

All lymph nodes were invaded by EPC with presence of capsular disruption and focal invasion of the resection margins.

A resected skin nodule also showed metastatic EPC, invading dermis and subcutaneous fat tissue (maximum depth $4 \mathrm{~mm}$ ) and displaying extensive lymphovascular invasion.

Because of the unfavourable characteristics (focal invasion of the resection margins, extensive lymphovascular invasion and invasion of the lymph nodes with capsular extension) the patient was referred for adjuvant radiotherapy. MRI of the axilla helped in delineating the target volume and identifying the brachial plexus. To obtain maximal control, a dose of $66 \mathrm{~Gy}$ in 33 sessions of 2 Gy was prescribed, using intensity modulated external beam radiotherapy. After 25 sessions, a small region of moist desquamation was observed deep in the axillary armpit. This was successfully treated with Mepilex. Overall, the treatment was well tolerated, and radiotherapy could be completed without any further problems (Figure 1, right).

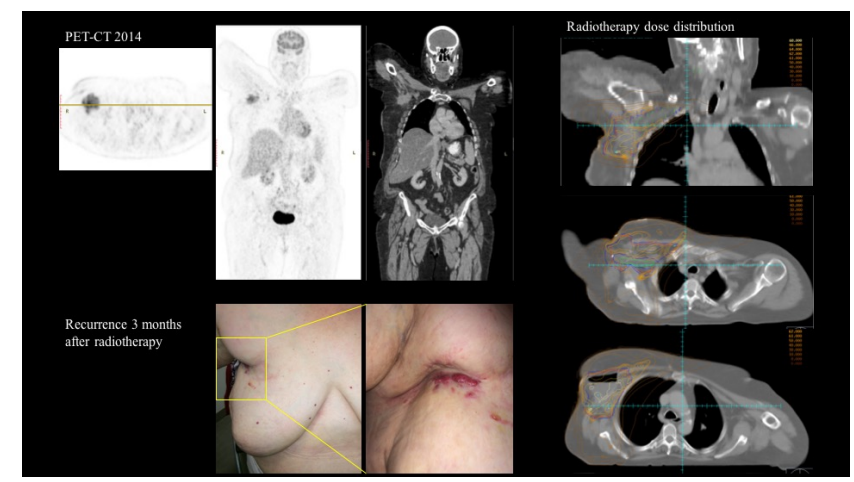

Figure 1 Iconography and clinical images of the case reported. Left above: PET-CT, after removal of the recurred EPC, revealing an axillary location of a suspicious mass. Right-side is the dosimetric planning of adjuvant radiotherapy after removal of the axillary mass. Left below is a photograph of the patient, showing in-field recurrence 3 months after removal of the axillary mass and adjuvant radiotherapy.
Unfortunately, three months after radiotherapy, a painful recurrence of the skin nodules was observed at the level of the breast scar, on the right scapula and at the dorsal side of the right upper arm (Figure 2). In order to alleviate symptoms, a wide resection of these metastases was performed. As expected, histopathological examination confirmed EPC recurrence.

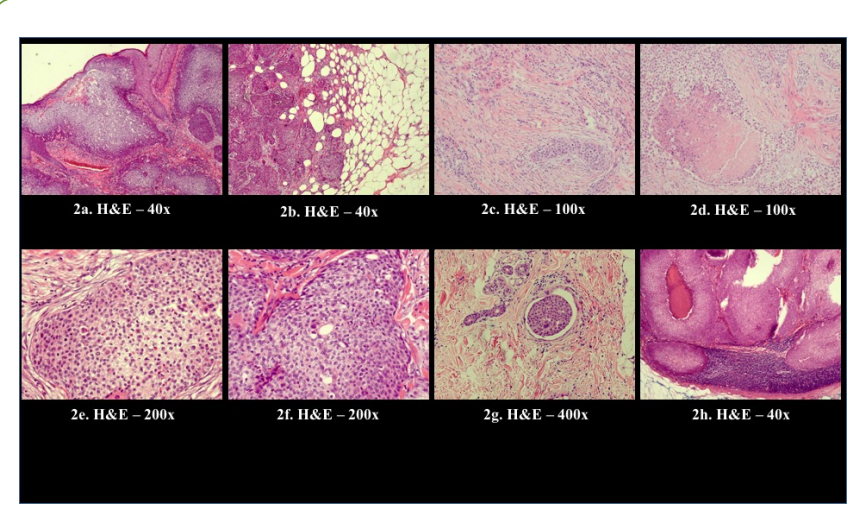

Figure 2 Histopathology of eccrine porocarcinoma. Histology of an infiltrative tumour partially connected to the epidermis (2a) (H\&E, original magnification 40x). Infiltrative growth pattern with invasion of the deep reticular dermis and subcutis ( $2 b$ and $2 c$ ) ( $H \& E$, original magnification $40 x$ and 100x). Note comedonecrosis (2d) (H\&E, original magnification 100x). Higher magnification reveals the presence of atypical basaloid tumour cells with pleomorphism and mitotic activity (2e) (H\&E, original magnification 200x). Focal clear cell change and ductal differentiation are seen in the tumornests (2f) (H\&E, original magnification 200x). Lymphatic invasion by neoplastic cells $(2 \mathrm{~g})$ (H\&E, original magnification 400x). Lymph node metastasis ( $2 \mathrm{~h})$ ( $\mathrm{H} \& \mathrm{E}$, original magnification 40x).

Because of limited evidence for chemotherapy in a patient already compromised by advanced age and multi-comorbidity (including diabetes, thrombo-embolic events and obesity) it was decided to withhold systemic therapy. When new skin metastases appeared, the possibility of palliative radiotherapy was proposed, but refused by the patient. It was decided to limit further therapy to best supportive care. Six months later the patient died from acute multi-organ failure.

Overall, 3.3 years elapsed between first resection of the primary tumour and death. The first recurrence was observed 22.5 months after a limited resection. Following extensive surgery and adjuvant radiotherapy for advanced loco-regional disease, the patient survived a further 17.5 months (Table 1).

Table 1 Histologic subtypes.

\begin{tabular}{|l|l|}
\hline Pushing subtype & Polypoid with a clear lower dermal limit \\
\hline Infiltrative subtype & Ill-defined lower limit with malignant clusters infiltrating in the dermis and/or hypodermis \\
\hline Pagetoid subtype & Intra-epidermal spreading of tumour cells on the side of the main tumour, mimicking Paget disease. \\
\hline
\end{tabular}




\section{Prevalence}

Eccrine porocarcinoma is an invasive carcinoma of the eccrine sweat glands, with an acrosyringeal origin. Other names were epidermotropic eccrine carcinoma, poroepithelioma, malignant hidroacanthoma simplex, malignant intraepidermal eccrine poroma, malignant eccrine poroma, malignant syringo-acanthoma and dysplastic poroma. In 1992, Snow et al. proposed a uniform terminology, to avoid further underreporting: the term eccrine porocarcinoma (EPC) was suggested [2].

Benign lesions (eccrine and more rarely, apocrine poroma) occur in up to $0.1 \%$ of primary cutaneous lesions [3], EPC constitutes only $0.005 \%$ to $0.01 \%$ of all skin cancers [4].

Mc Lean et al. divided sweat gland carcinomas in 7 subgroups, with the classical EPC (or eccrine adenocarcinoma) being the most prevalent [5]:

- Classic-type eccrine adenocarcinoma (or eccrine porocarcinoma)

- Syringoid eccrine carcinoma

- Microcystic Adnexal Carcinoma

- Mucinous eccrine carcinoma

- Muco-epidermoid carcinoma

- Adenoid cystic carcinoma

- Aggressive digital papillary adenoma/adenocarcinoma

EPC can arise de novo or evolve from a benign poroma, an evolution described to occur in one-fifth of poromas [6]. However, poroma is primarily found in regions with a high density of eccrine sweat glands (palms or soles), whereas porocarcinoma occurs only rarely in these regions and more frequently on the extremities ( $>50 \%$ ), the trunk, the head and neck. Other locations have been described (breast, vulva, scrotum, nail bed).

EPC occurs more often in the sixth to seventh decade of life, with a modestly higher prevalence in women. A correlation might exist with immuno-depression (transplant, HIV, chemotherapy), radiation exposure and chronic radiation dermatitis [7]. It has been described as developing in association with extra mammary Paget's disease [8], sarcoidosis [9], chronic lymphocytic leukaemia [10], pernicious anaemia [11], Hodgkin's disease [8], nevus sebaceous [12], HIV [13] and xeroderma pigmentosum [14].

After proliferation within the epidermis, invasion of papillary dermis and reticular dermis follow. After invasion of the dermal lymphatics, the tumour can spread to the lymph nodes (described in $20 \%$ of cases), in distant solid organs $(10 \%$ of cases) or recur locally (20\% of cases) [15].

\section{Clinical Diagnosis}

EPCs are not clinically distinctive and mostly described as verrucous plaques or polypoid growths, often clinically misdiagnosed as squamous cell carcinoma, Bowen's disease, seborrheic keratosis or pyogenic granuloma. The lesions can be erythematous to violaceous in colour. Porocarcinoma has a tendency to be more exophytic and ulcerative compared to poroma [16] (Figure 3). Biopsy is mandatory for a correct diagnosis.

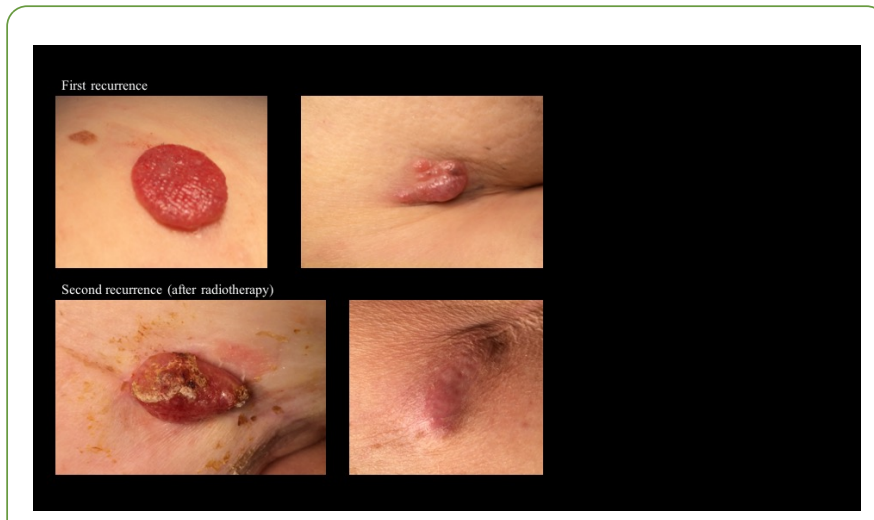

Figure 3 Clinical aspect clinical aspect of tumour: above: first recurrence; below: second recurrence, 3 months after radiotherapy.

\section{Work up}

Work up should consist of an extensive medical history, including toxic exposure, extensive clinical evaluation of the skin and adjacent lymph node regions. CT of pelvis, abdomen and thorax should be performed. Bone scan is useful to exclude bone metastases.

PET-CT for porocarcinoma seems promising: in 2005 Cho describes the case of a recurrent porocarcinoma, where FDGPET-CT clearly highlighted multiple FDG-avid foci over skin and lymph nodes [17]. Histology confirmed the diagnosis. A second FDG-PET-CT five months later revealed lesions in skin, bone, lungs and lymph nodes. Another case was reported in 2007, also confirming correct diagnosis of metastases using FDG-PETCT [18] and a similar third case was described in 2014 by Golemi et al. [19]. The primary tumour as well as subsequent metastases display a high glucose metabolism, which probably explains adequate detection.

\section{Histology}

The clinical and histologic features of eccrine poroma, porocarcinoma and related lesions are thoroughly described in a review by Sawaya et al. [20]. 
Histopathologically, the major differential diagnoses are eccrine poroma, hidradenoma, hidradenocarcinoma, squamous cell carcinoma and basal cell carcinoma.

Especially in superficial (shave) biopsies eccrine poroma can be difficult to differentiate from non-invasive (in situ) EPC or pushing subtype of EPC. However, eccrine poroma lacks dermal invasion, significant cytologic atypia and necrosis.

Most cases of EPC are easily distinguishable from basal cell carcinomas, however EPC must be differentiated from infiltrating subtypes of basal cell carcinomas by the presence of intercellular bridges and the absence of peripheral palisading and tumour-tumour retraction artefact. Squamoid variants of EPC may be difficult to differentiate from squamous cell carcinoma but squamous cell carcinoma lacks ductal

Table 2 Differential diagnoses. structures and intracytoplasmic lumina, which can be highlighted with the immunohistochemical EMA and CEA staining. In this particular case, an invasive breast ductal carcinoma can be added in the differential diagnosis, as an EPC presenting in the axillary lymph nodes or in the breast is hard to differentiate from a primary breast carcinoma. Histology is complex, and misdiagnosis has been reported in up to $1 / 3$ of cases [21]. Based on the morphology (connection of the tumour to the epidermis and the ductal eccrine differentiation) and the immunohistochemistry (negativity of CK7, ER, PR and GATA3) of the present case, the diagnosis of a primary breast carcinoma was unlikely. Moreover, the diffuse tumour-positivity for p63 and CK5 conflicts with a metastatic tumour to the skin, which is typically negative for p63 and CK5 (Table 2).

\begin{tabular}{|c|c|}
\hline \multicolumn{2}{|c|}{ Clinical and histopathological differential diagnosis (DD) } \\
\hline Eccrine Poroma & Porocarcinoma \\
\hline Basal cell carcinoma & Basal cell carcinoma \\
\hline Seborrheic keratosis & Seborrheic keratosis (clinical DD only) \\
\hline Hidradenoma & Hidradenoma \\
\hline Pyogenic granuloma (clinical DD only) & Pyogenic granuloma (clinical DD only) \\
\hline Acrochordons (clinical DD only) & Acrochordons (clinical DD only) \\
\hline Verrucae & Verrucae \\
\hline Naevus (clinical DD only) & Hidradenocarcinoma \\
\hline \multirow[t]{6}{*}{ Fibroma (clinical DD only) } & Bowen's disease (SCC in situ) \\
\hline & Paget's disease \\
\hline & Amelanotic melanoma (clinical DD only) \\
\hline & Squamous cell carcinoma \\
\hline & Metastasis (lung cancer, other) \\
\hline & Invasive ductal cell breast carcinoma \\
\hline
\end{tabular}

\section{Prognostic factors}

In porocarcinoma, tumour size is of no prognostic value. Unfavourable prognostic indicators for EPC include mitotic activity, lympho-vascular invasion and tumour thickness. Tumours with an infiltrating rather than a "pushing" lower border is associated with a greater risk of recurrence $[22,23]$.

In a retrospective analysis of 24 patients, EPC was divided in three subtypes: infiltrative, pushing and pagetoid [21]. Recurrence occurred in $35 \%$ of patients and was associated with the histological subtypes displaying pagetoid margins $(2 / 2)$ or infiltrative margins (4/10), while none of the "pushing" subtypes relapsed. Similar to the findings of Robson et al. an infiltrative tumour margin was predictive for local recurrence [6]. No effect of the extent of resection margin was observed ( $\geq 2 \mathrm{~cm}$ ( 6 pts.) , $<2 \mathrm{~cm}$ (13 pts.) or narrow margin by modified/slow Mohs micrographic surgery (MMS) (2 pts.) were compared), whatever the subtype. The authors suggested narrow-margin excision for the less aggressive pushing EPC and modified MMS for the infiltrative or pagetoid subtypes.

\section{Treatment}

After biopsy and work up, the primary treatment for both the benign poroma and its malignant counterpart, is surgery.

\section{Non-invasive poroma}

Benign poroma should always be removed, since $20 \%$ risk transition into its invasive counterpart $[6,24]$. Removal results in a high probability of complete curation, even after simple excision for small lesions or MMS for large or deep lesions. For superficial lesions fulguration, cautery, excision or electrocauterization procedures are sufficient. 


\section{Invasive porocarcinoma}

Surgery with wide excision is the standard treatment for non-metastatic EPC. In early stage porocarcinoma, wide surgical excision results in a high cure rate of $70-80 \%$. Lymphadenectomy is advised in cases of poor prognostic characteristics (lymphovascular invasion, infiltrative or pagetoid tumour margin or high-grade lesions) because of its high tendency for local recurrence (in 20\% of cases), locoregional recurrence ( $20 \%$ of cases) and visceral metastasis (10\% of cases) [6,25-27]. Little evidence exists on the benefit of adjuvant treatment, but in case of positive resection margins, adjuvant radiotherapy seems advisable. No clear guidelines on dose or fractionation are available.

In case of loco-regional recurrence, re-excision remains the treatment paradigm. Radiotherapy and chemotherapy are also options.

Metastatic disease survival can be prolonged with palliative chemotherapy and/or radiotherapy and in case of hormonal receptor positivity, Tamoxifen. However, outcomes are poor, with survival varying between 5 and 24 months. An overview on treatment options is listed in Table 3 [28-46].

Table 3 Systemic treatment.

\begin{tabular}{|c|c|c|}
\hline Cyclophosphamide, bleomycine, cisplatin, 5-FU & 24 pt, PR - 5-8 months & Coonly [28] \\
\hline Melphalan \& Intra-arterial 5-FU & $1 \mathrm{pt}, \mathrm{CR}$ & Briscoe [29] \\
\hline 5-FU + radiotherapy & $1 \mathrm{pt}, \mathrm{PR}$ & Mitts [30] \\
\hline 5-FU continuous ( $4 \mathrm{~d} 1 \mathrm{q} 4)$ & -- & Swanson [31] \\
\hline $\begin{array}{l}\text { Doxorubicine, Mitomycine-C, Vincristine, 5-FU } \\
\text { alternating with Cisplatinum, Bleomycin (1q2) }\end{array}$ & $\begin{array}{l}\text { Prolonged CR } \\
\text { (but serious toxicity after } 9 \text { cycli) - } 16 \text { months }\end{array}$ & Piedbois \\
\hline Cyclophosphamide, vincristine, prednisone & PR, 2-4 months & Coonly (28) \\
\hline \multirow[t]{2}{*}{ Isotretinoin } & $1 \mathrm{pt}, \mathrm{PR}-8 \mathrm{wks}$ & Roach [8] \\
\hline & $1 \mathrm{pt}$, no response & Friedland [32] \\
\hline \multirow[t]{2}{*}{ Interferon-Alpha, Interleukin-2 } & $1 \mathrm{pt}, \mathrm{SD}$ & Dummer [33] \\
\hline & $1 \mathrm{pt}, \mathrm{SD}$ & Barzi [34] \\
\hline \multirow[t]{2}{*}{ Docetaxel } & $1 \mathrm{pt}, \mathrm{PR}$ & Plunkett [35] \\
\hline & CR in lung, $P R$ in breast & Aaribi [36] \\
\hline Docetaxel, topic 5-FU & 1 pt, CR 2 y & De Bree [37] \\
\hline Paclitaxel, interferon alpha & $1 \mathrm{pt}, \mathrm{SD}$ & Gutermuth [38] \\
\hline \multirow[t]{2}{*}{ 5-fluorouracil } & $1 \mathrm{pt}, \mathrm{CR}$ & Bellman [39] \\
\hline & $1 \mathrm{pt}, \mathrm{CR}$ & Swanson [31] \\
\hline 5-Fluorouracil, Epirubicin, Cyclophosphamide & $1 \mathrm{pt}, \mathrm{SD}-17$ months & Mc Lean [5] \\
\hline \multirow[t]{4}{*}{ Tamoxifen } & 1 pt, CR 3 years & Schroder [40] \\
\hline & $2 \mathrm{pt}$ & Shridar [41] \\
\hline & CR - 3 years in ER/PR positive tumor & \\
\hline & No response in ER/PR negative & \\
\hline Topical diphencyprone 6\% & $1 \mathrm{pt}, \mathrm{CR}$ (skin lesions) 6 months & Kutty [42] \\
\hline \multirow[t]{4}{*}{ Radiotherapy } & IMRT $30 \times 2$ Gy - DFS 10 months & Zeidan [43] \\
\hline & -- & Mertens [44] \\
\hline & $1 \mathrm{pt}, \mathrm{LC} 18$ months & Grizzard [45] \\
\hline & 1 pt, Cyberknife, $10 \times 5$ Gy, CR 1 year & Fujimura [46] \\
\hline
\end{tabular}

Abbreviations: CR: Complete Remission; PR: Partial Remission; SD: Stable Disease; LC: Local Control; Pt: Patient; ER: Estrogen Receptor; PR: Progesterone Receptor

\section{Discussion}

In this patient, the initial clinical presentation of a superficial polypoid breast nodule might have suggested a non-breast tumour. Unfortunately, no images of the primary lesion exist.
No detailed information on the histopathology of the excisional biopsy could be found. Information on prognostic characteristics, including infiltrative spread, presence of lymphovascular invasion and mitotic activity, could have helped differentiating between a conservative and a more invasive approach. A postoperative work-up with CT-thorax, 
CT-abdomen and bone scan at initial diagnosis would have been justified, completed with PET-CT in case of doubt or suspicion for metastases. Even though initial clinical axillary examination and ultrasonography were negative, lymphadenectomy is advisable in case of unfavourable tumour characteristics.

No evidence exists on adjuvant treatment after surgery. However, radiotherapy had been an option in case of positive resection margins or extensive lympho-vascular invasion.

At the moment of recurrence, resection of the superficial lesions was performed without preoperative staging, overlooking a large axillary mass and necessitating a second surgical intervention in a patient, already compromised by age and co-morbidity. As postoperative staging revealed residual tumor, and as patient and family choose for maximal locoregional control, salvage treatment with debulking and adjuvant radiotherapy were performed. Because of the proximity of the brachial plexus a normofractionated schedule was opted for. Evidence on hypofractionation for EPC is lacking; in retrospect however, a lower number of treatment sessions to reduce the burden of transportation for patient and family might have been an acceptable alternative. Even in the proximity of the brachial plexus, a schedule consisting of 20 fractions of $2.67 \mathrm{~Gy}$ or 5 fractions of $6 \mathrm{~Gy}$ may have been safe, as these results respectively in an EQD2 of 63.6 Gy and $64.3 \mathrm{~Gy}$ when applying an $\alpha / \beta$ of $1.5 \mathrm{~Gy}$. As yet, no evidence exists on the benefit of hypofractionation for EPC.

No systemic therapy was added: as hormone receptors were negative, no benefit was expected from adding tamoxifen. At the time of second relapse, the performance status of this patient, already compromised by morbid obesity and diabetes, did not permit administration of docetaxel or other chemotherapy.

\section{Conclusion}

Breast locations of EPC, an uncommon skin cancer, are misleading and could cause surgeons to underestimate the risks of lymphatic and dermal spread of this type of carcinoma, which behaves quite differently from breast carcinoma. Various appearances and a broad range of differential diagnoses are prone to misdiagnosis. If complete surgical excision is possible in early stage disease, loco-regional control can be accomplished in $70-80 \%$ of cases. Lymphadenectomy is recommended in case of unfavourable prognostic characteristics. Evidence on the benefit of adjuvant treatment is lacking. However, there may be a role for radiotherapy in case of positive resection margins or extensive lymphovascular spread.

Though prolonged disease stabilization in metastatic disease is reported with poly-chemotherapy, it comes at the cost of severe toxicity [27]. Reports exist on complete or partial remission following the use of docetaxel or isotretinoin. In ER/PR positive tumours, use of tamoxifen may stabilize disease.
In brief, the presented case is an example of an apparently innocuous breast nodule, where initial underestimation of the aggressiveness of EPC histology may have compromised the final outcome.

\section{Acknowledgements}

We gratefully thank Pauline Page-Jones for her textual revision of the manuscript.

\section{Ethical Approval}

All procedures performed in studies involving human participants were in accordance with the ethical standards of the institutional and/or national research committee and with the 1964 Helsinki declaration and its later amendments or comparable ethical standards. Informed consent for publication of the case was obtained.

\section{Conflict of Interest}

Author C. Monten declares that she has no conflict of interest. Author J. Berwouts declares that she has no conflict of interest. Author L. Veldeman declares that she has no conflict of interest. Author D. Creytens declares that he has no conflict of interest. Author G. Braems declares that he has no conflict of interest.

\section{References}

1. Calcagni $M L$, Taralli $S$, Cardillo $G$, Graziano $P$, lalongo $P$, et al. (2016) Diagnostic performance of (18) f-fluorodeoxyglucose in 162 small pulmonary nodules incidentally detected in subjects without a history of malignancy. Ann Thorac Surg 101: 1303-1309.

2. Snow SN, Reizner GT (1992) Mucinous eccrine carcinoma of the eyelid. Cancer 70: 2099-2104.

3. Pylyser K, De Wolf-Peeters C, Marien K (1983) The histology of eccrine poromas: A study of 14 cases. Dermatologica 167: 243-249.

4. Mehregan AH, Hashimoto K, Rahbari H (1983) Eccrine adenocarcinoma. A clinicopathologic study of 35 cases. Arch Dermatol 119: 104-114.

5. McLean SR, Shousha S, Francis N, Lim A, Eccles S, et al. (2007) Metastatic ductal eccrine adenocarcinoma masquerading as an invasive ductal carcinoma of the male breast. J Cutan Pathol 34: 934-938.

6. Robson A, Greene J, Ansari N, Kim B, Seed PT, et al. (2001) Eccrine porocarcinoma (malignant eccrine poroma): $A$ clinicopathologic study of 69 cases. Am J Surg Pathol 25: 710-20.

7. Requena L, Marquina A, Alegre V, Aliaga A, Sanchez Yus E (1990) Sclerosing-sweat-duct (microcystic adnexal) carcinoma--a tumor from a single eccrine origin. Clin Exp Dermatol 15: 222-224.

8. Roach M (1983) A malignant eccrine poroma responds to isotretinoin (13-cis-retinoic acid). Ann Intern Med 99: 486-468.

9. Bottles K, Sagebiel RW, McNutt NS, Jensen B, Deveney K (1984) Malignant eccrine poroma. Case report and review of the literature. Cancer 53: 1579-1585. 
10. Claudy AL, Garcier F, Kanitakis J (1984) Eccrine porocarcinoma. Ultrastructural and immunological study. J Dermatol 11: 282-286.

11. Puttick L, Ince P, Comaish JS (1986) Three cases of eccrine porocarcinoma. Br J Dermatol 115: 111-116.

12. Tarkhan, Domingo J (1985) Metastasizing eccrine porocarcinoma developing in a sebaceous nevus of Jadassohn. Report of a case. Arch Dermatol 121: 413-415.

13. Mahomed F, Blok J, Grayson W (2008) The squamous variant of eccrine porocarcinoma: A clinicopathological study of 21 cases. J Clin Pathol 61: 361-365.

14. Poiares Baptista A, Tellechea O, Reis JP, Cunha MF, Figueiredo P (1993) Eccrine porocarcinoma. A review of 24 cases]. Ann Dermatol Venereol 120: 107-115.

15. Shiohara J, Koga H, Uhara H, Takata M, Saida T (2007) Eccrine porocarcinoma: Clinical and pathological studies of 12 cases. J Dermatol 34: 516-522.

16. Brown CW Jr., Dy LC (2008) Eccrine porocarcinoma. Dermatol Ther 21: 433-438.

17. Cho SB, Roh MR, Yun M, Yun SK, Lee MG, et al. (2005) (18)Ffluorodeoxyglucose positron emission tomography detection of eccrine porocarcinoma. Br J Dermatol 152: 372-373.

18. Li SH, Chen MT, Chen YJ, Wang SL, Chang-Yu W (2007) Metastatic eccrine porocarcinoma detected on FDG PET/CT. Clin Nucl Med 32: 743-745.

19. Golemi A, Hanspeter E, Brugger E, Ruiu A, Farsad M (2014) FDG $\mathrm{PET} / \mathrm{CT}$ in malignant eccrine porocarcinoma arising in a preexisting poroma. Clin Nucl Med 39: 456-458.

20. Sawaya JL, Khachemoune A. Poroma: A review of eccrine, apocrine, and malignant forms. Int J Dermatol 53: 1053-1061.

21. Belin E, Ezzedine K, Stanislas S, Lalanne N, Beylot-Barry M, et al. (2011) Factors in the surgical management of primary eccrine porocarcinoma: prognostic histological factors can guide the surgical procedure. Br J Dermatol 165: 985-989.

22. Skowron F, Poulhalon N, Balme B, Touzet S, Thomas L (2014) Primary eccrine porocarcinoma: A clinicopathological study of 50 cases. Ann Dermatol Venereol 141: 258-264.

23. Riera-Leal L, Guevara-Gutierrez E, Barrientos-Garcia JG, Madrigal-Kasem R, Briseno-Rodriguez G, et al. (2015) Eccrine porocarcinoma: Epidemiologic and histopathologic characteristics. Int J Dermatol 54: 580-586.

24. Shaw M, McKee PH, Lowe D, Black MM (1982) Malignant eccrine poroma: A study of twenty-seven cases. Br J Dermatol 107: $675-680$

25. Vleugels FR, Girouard SD, Schmults CD, Ng AK, Russell SE, et al. (2012) Metastatic eccrine porocarcinoma after Mohs micrographic surgery: a case report. Journal of clinical oncology: Official Journal of the American Society of Clinical Oncology 30: e188-191.

26. Luz Mde A, Ogata DC, Montenegro MF, Biasi LJ, Ribeiro LC (2010) Eccrine porocarcinoma (malignant eccrine poroma): A series of eight challenging cases. Clinics 65: 739-742.

27. Piedbois P, Breau JL, Morere JF, Israel L (1987) Sweat gland carcinoma with bone and visceral metastases. Prolonged complete remission lasting 16 months as a result of chemotherapy. Cancer 60: 170-172.
28. Coonley CJ, Schauer P, Kelsen DP, Sordillo P, Huvos AG (1985) Chemotherapy of metastatic sweat gland carcinoma. A retrospective review. AJCO 8: 307-311.

29. Briscoe KE, Grage T, Kennedy BJ (1978) Sustained complete remission of metastatic sweat gland carcinoma following regional hyperthermic perfusion. JAMA 240: 51-52.

30. Mitts DL, Smith MT, Russell L, Bannayan GA, Cruz AB Jr. (1976) Sweat gland carcinoma: A clinico-pathological reappraisal. J Surg Oncol 8: 23-29.

31. Swanson JD Jr., Pazdur R, Sykes E (1989) Metastatic sweat gland carcinoma: Response to 5-fluorouracil infusion. J Surg Oncol 42: 69-72.

32. Friedland M, Bajracharya A, Arlin Z (1984) Malignant eccrine poroma and isotretinoin. Ann Intern Med 100: 614.

33. Dummer R, Becker JC, Boser B, Hartmann AA, Burg G (1992) Successful therapy of metastatic eccrine poroma using perilesional interferon alfa and interleukin 2. Arch Dermatol 128: 1127-1128.

34. Barzi AS, Ruggeri S, Recchia F, Bertoldi I (1997) Malignant metastatic eccrine poroma. Proposal for a new therapeutic protocol. Dermatol Surg 23: 267-272.

35. Plunkett TA, Hanby AM, Miles DW, Rubens RD (2001) Metastatic eccrine porocarcinoma: Response to docetaxel (Taxotere) chemotherapy. ESMO 12: 411-414.

36. Aaribi I, Mohtaram A, Ameur El-Youbi MB, Kharmoum J, ElKabous M, et al. (2013) Successful management of metastatic eccrine porocarcinoma. Case Rep Oncol Med 2013: 282536.

37. De Bree E, Volalakis E, Tsetis D, Varthalitis $Y$, Panagiotidis J, et al. (2005) Treatment of advanced malignant eccrine poroma with locoregional chemotherapy. Br J Dermatol 152: 1051-1055.

38. Gutermuth J, Audring H, Voit C, Trefzer U, Haas N (2004) Antitumour activity of paclitaxel and interferon-alpha in a case of metastatic eccrine porocarcinoma. J Eur Acad Dermatol Venereol 18: 477-479.

39. Bellman B, Gregory NA, Silvers D, Fountain KS (1995) Sweat gland carcinoma with metastases to the skin: Response to 5fluorouracil chemotherapy. Cutis 55: 221-224.

40. Schroder U, Dries V, Klussmann JP, Wittekindt C, Eckel HE (2004) Successful adjuvant tamoxifen therapy for estrogen receptorpositive metastasizing sweat gland adenocarcinoma: need for a clinical trial? Ann Otol Rhinol Laryngol 113: 242-244.

41. Sridhar KS, Benedetto P, Otrakji CL, Charyulu KK (1989) Response of eccrine adenocarcinoma to tamoxifen. Cancer 64: 366-370.

42. Kutty A, Harper F, Akhras V (2013) Treatment of multiple bilateral primary eccrine porocarcinoma with topical diphencyprone: a case report. Br J Dermatol 169: 1159-1661.

43. Zeidan YH, Zauls AJ, Bilic M, Lentsch EJ, Sharma AK (2010) Treatment of eccrine porocarcinoma with metastasis to the parotid gland using intensity-modulated radiation therapy: a case report. J Med Case Rep 4: 147.

44. Mertens WC, Shum DT, Gilchrist JA (1996) Adenocarcinoma of the eccrine sweat gland: response to both combination chemotherapy and local field irradiation. European journal of cancer 32A: 372-373.

45. Grizzard WS, Torezynski E, Edwards WC (1976) Adenocarcinoma of eccrine sweat glands. Arch Ophthalmol 94: 2119-2123. 
46. Fujimura $T$, Hashimoto A, Furudate $S$, Kambayashi $Y$, Haga $T$, et al. (2014) Successful Treatment of Eccrine Porocarcinoma
Metastasized to a Cervical Lymph Node with CyberKnife Radiosurgery. Case Rep Dermatol 6: 159-163. 